\title{
On Normalish subgroups of the R. Thompson groups $^{\star}$
}

\author{
Collin Bleak ${ }^{1[0000-0001-5790-1940]}$ \\ University of St Andrews, St Andrews, Scotland, UK, KY16 9SS \\ cb211@st-andrews.ac.uk http://www-groups.mcs.st-and.ac.uk/ collin/
}

\begin{abstract}
Results in $C^{*}$ algebras, of Matte Bon and Le Boudec, and of Haagerup and Olesen, apply to the R. Thompson groups $F \leq T \leq V$. These results together show that $F$ is non-amenable if and only if $T$ has a simple reduced $C^{*}$-algebra.

In further investigations into the structure of $C^{*}$-algebras, Breuillard, Kalantar, Kennedy, and Ozawa introduce the notion of a normalish subgroup of a group $G$. They show that if a group $G$ admits no non-trivial finite normal subgroups and no normalish amenable subgroups then it has a simple reduced $C^{*}$-algebra. Our chief result concerns the R. Thompson groups $F<T<V$; we show that there is an elementary amenable group $E<F($ where here, $E \cong \ldots)(\mathbb{Z})(\mathbb{Z})(\mathbb{Z})$ with $E$ normalish in $V$.

The proof given uses a natural partial action of the group $V$ on a regular language determined by a synchronizing automaton in order to verify a certain stability condition: once again highlighting the existence of interesting intersections of the theory of $V$ with various forms of formal language theory.
\end{abstract}

Keywords: Thompson's group · amenable · $C^{*}$-simplicity · regular language ' synchronizing automata ' group actions ' normalish subgroups $\cdot$ wreath product.

\section{Introduction}

In this note we show that for the R. Thompson groups $F \leq T \leq V$ there is an elementary amenable group $E \leq F$ so that $E$ is normalish in each of the groups $F, T$, and $V$.

\subsection{General motivating background}

Various weakenings of the notion of normal subgroup were introduced between 2014 and 2018 in order to obtain insight into the $C^{*}$-simplicity of the (reduced) group algebra $C_{r}^{*}(G)$ of a group $G$. This has had particular impact for infinite simple groups such as the R. Thompson groups $T$ and $V$. The concept of a normalish subgroup of a group was introduced by in the seminal paper of Breuilliard,

* The author wishes to gratefully acknowledge support from the EPSRC grant $\mathrm{EP} / \mathrm{R} 032866 / 1$. 
Kalantar, Kennedy, and Ozawa [5]. They show that a discrete group $G$ with no non-trivial finite normal subgroups and no amenable normalish subgroups is $C^{*}$ simple. In that paper, they also obtain the just-previously-announced result of Haagerup and Olesen [8] that if the reduced group $C^{*}$-algebra $C_{r}^{*}(T)$ is simple, then $F$ is non-amenable.

Meanwhile, Kennedy in [9] shows that a countable group $G$ is $C^{*}$-simple (has simple reduced $C^{*}$-algebra) if and only if $G$ admits no non-trivial amenable URS (uniformly recurrent subgroup). Using this, Le Boudec and Matte Bon in [10] show the converse of the stated Haagerup-Olesen result, if $F$ is non-amenable, then the reduced $C^{*}$-algebra of $T$ must be simple.

Indeed, for those interested in the question of the non-amenability of the R. Thompson group $F$, the focus has passed through the exploration of the uniformly recurrent subgroups of $T$ to understanding the point stabilisers of the action of $T$ on its Furstenberg boundary. Here, there are two possible cases, and $F$ will be non-amenable precisely if these point stabilisers are trivial (see [10]). Despite this shift, we find the concept of normalish subgroups of simple groups like $F$ and $T$ to be of interest, and that is the focus of this note.

\subsection{Core results}

Let $G \leq H$ be groups. The group $G$ is normalish in $H$ if for any finite set of elements $\left\{c_{1}, c_{2}, \ldots, c_{k}\right\}$ the intersection

$$
\bigcap_{i=1}^{k} G^{c_{i}}
$$

is infinite.

Our chief result is the following:

Theorem 1 There is an embedding of the elementary amenable group

$$
\infty(\mathbb{Z} \succ \mathbb{Z})=\ldots(\mathbb{Z})(\mathbb{Z}) \succ \mathbb{Z}
$$

into $R$. Thompson's group $F$ so that the image group $E$ is normalish in $V$.

Observe the corollary that $E$ is then an amenable normalish subgroup of both $F$ and of $T$ as well.

\subsection{Specific history of the core result}

We should mention some other history related to this result. In [1] we showed the existence of an infinite direct sum of copies of $\mathbb{Z}$ that could be found embedded as a normalish amenable subgroup of $F$, and discussed our conjecture (disproven here) that any normalish amenable subgroup of $T$ should either contain an embedded subgroup isomorphic to R. Thompson's group $F$ or to a non-abelian free subgroup. Meanwhile, the paper [10] shows that $V$ contains an amenable 
normalish torsion group $\Lambda$ : the subgroup of $V$ consisting of those elements which are automorphisms of the infinite rooted binary tree $\mathcal{T}_{2}$. These automorphism arise as finite compositions of the tree automorphisms that swap the two child vertices of any particular vertex (copying the dependent trees identically). The group $\Lambda$ is normalish for reasons that are very similar to why our own group $E$ is normalish, and it is a limit of finite groups hence elementary amenable. However, the group $\Lambda$ is not a subgroup of $F$ nor of $T$.

\subsection{An unexpected visitor: a controlling synchronizing automaton}

A note on the proof: for experts on R. Thompson groups, the embedded copy of $E$ that we find will clearly be normalish in $V$ after short inspection. However, the technical proof of this requires a bit of work in that the conjugation action on our generators needs to not introduce too many breakpoints into our group elements, and also in that we need to have enough group elements that the set is essentially closed under translations by arbitrary elements in $V$. The second task is the harder one if we are to avoid having further subgroups isomorphic to R. Thompson's group $F$. We approach this by introducing a partial action of $V$ on a regular language which is determined by a synchronizing automaton. We link this to the action of $V$ on an infinite specified subset of $E$. By considering our partial action on the regular language, we can show there is an infinite subset of $E$ that is not moved off of itself too much under the action of finitely many elements of $V$.

Thanks:

We would like to thank Adrienne Le Boudec for kind and informative conversations where he has helped the author of the present note to understand some of the amazing events that have transpired in the field of $C^{*}$-algebras over the last six years.

\section{The interval and the circle as quotients of Cantor space, and some related language}

Let $I:=[0,1] \subset \mathbb{R}$ represent the unit interval in the real numbers. Let $\mathfrak{C}:=$ $\{0,1\}^{\omega}$ represent the Cantor space that arises as the infinite cartesian product of the discrete space $\{0,1\}$ with itself, with the product indexed by the ordinal $\omega$. As we will act on our Cantor space from the right via prefix substitutions, we will express elements of Cantor space as left infinite strings, so a typical element $\overleftarrow{x}$ of $\mathfrak{C}$ will be written as $\overleftarrow{x}=\ldots x_{2} x_{1} x_{0}$ where each $x_{i}$ is either a 0 or a 1 . Note that in this usage, and for such left-infinite strings, we will refer to any finite rightmost contiguous substring as a prefix of the infinite string (and we will use the word prefix in this way as well when comparing finite strings, which we will formalise below). The monoid $\{0,1\}^{*}$ of finite strings under the concatenation operator "^" (e.g., 00110^1001 =001101001) will be central to our analysis and we might refer to an element of $\{0,1\}^{*}$ as an address, for reasons which will become clear. 
We give the monoid of finite words $\{0,1\}^{*}$ the prefix-based partial ordering as follows: if $p_{1}, p_{2} \in\{0,1\}^{*}$ with $p_{1}=x_{j} x_{j-1} \ldots x_{1} x_{0}$ and $p_{2}=$ $y_{k} y_{k-1} \ldots y_{1} y_{0}$ (where each $x_{i}$ and $y_{i}$ is in the set $\{0,1\}$ for each valid index $i$ ), we say $p_{1} \leq p_{2}$ if and only if $j \leq k$ and for all indices $0 \leq i \leq j$ we have $x_{i}=y_{i}$. Recall that with this partial ordering, a complete antichain $\mathcal{A}$ of $\{0,1\}^{*}$ is a finite set $\left\{p_{1}, p_{2}, \ldots, p_{k}\right\}$ so that for each pair of distinct indices $i$ and $j$ we have that $p_{i}$ and $p_{j}$ are incomparable (written $p_{1} \perp p_{2}$, and meaning that both $p_{1} \not \leq p_{2}$ and $p_{2} \not \leq p_{1}$ are true) and for any $w \in\{0,1\}^{*}$ we have some index $r$ so that either $w \leq p_{r}$ or $p_{r} \leq w$.

The monoid $\{0,1\}^{*}$ with the partial order above can be naturally drawn as a rooted infinite binary tree, with its vertices being the elements of $\{0,1\}^{*}$, and where we draw an edge from vertices $r$ to $s$ if $r \leq s$ and the length of $s$ (denoted $|s|)$ is one greater than the length of $r$. We will denote this tree as $\mathcal{T}_{2}$ and sketch a small neighbourhood of its root in the figure below (the tree $\mathcal{T}_{2}$ is often drawn so as to "open out" as one descends).

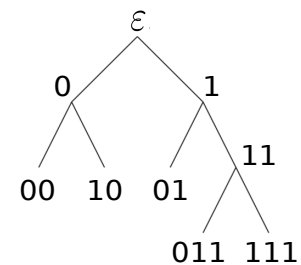

Fig. 1. A neighbourhood of the root $\varepsilon$ of the tree $\mathcal{T}_{2}$

For any finite word $w=w_{k} w_{k-1} \ldots w_{1} w_{0} \in\{0,1\}^{*}$ we obtain the basic open set $\mathfrak{C} w$ for the topology of $\mathfrak{C}$. Specifically, $\mathfrak{C} w$ is the set of all points in Cantor space with prefix $w$ :

$$
\mathfrak{C} w=\left\{\overleftarrow{x}^{\wedge} w: \overleftarrow{x} \in \mathfrak{C}\right\}
$$

We will refer to such basic open sets as cones, and for a given finite word $w \in$ $\{0,1\}^{*}$ the set $\mathfrak{C} w$ will be called the cone at (address) $w$. It is a standard fact that one can identify the Cantor space $\mathfrak{C}$ with the boundary of $\mathcal{T}_{2}$, or with the set of infinite descending paths in the tree (which correspond to infinite sequences of edge lables, if one labels each edge of $\mathcal{T}_{2}$ with a 0 or a 1 , depending on the letter of the extension connecting the shorter address to the longer address.)

Recall there is a standard quotient map $q: \mathfrak{C} \rightarrow[0,1]$, which we define fully here in order to give some practice with our right-to-left indexing notation. Let

$$
\overleftarrow{x}=\ldots x_{2} x_{1} x_{0} \in \mathfrak{C}
$$

We have 


$$
(\overleftarrow{x}) q:=\sum_{i=0}^{\infty} x_{i} \cdot \frac{1}{2^{i+1}}
$$

which we can think of as the ordinary map which interprets a real number in $[0,1]$ from its binary expansion.

We further recall that given any prefix $w=w_{k} w_{k-1} \ldots w_{1} w_{0}$ the map $q$ identifies the two points $\overline{1} 0 w_{k} w_{k-1} \ldots w_{1} w_{0}$ and $\overline{0} 1 w_{k} w_{k-1} \ldots w_{1} w_{0}$. The resulting two-point equivalence classes map onto the dyadic rationals in $\mathbb{Z}[1 / 2] \cap(0,1) \subset \mathbb{R}$, and further, the cone $\mathfrak{C} w$ at $w$ maps to the closed interval $\mathcal{I}_{w}$ of radius $(1 / 2)^{k+2}$ centered at the diadic point $d_{w}$ which is defined by the infinite sum

$$
d_{w}:=\sum_{i=0}^{\infty} w_{i} \cdot \frac{1}{2^{i+1}}
$$

where we set $w_{k+1}=1$ and $w_{m}=0$ for all $m>k+1$. For example, if $w=01$, then $k=1$ and we have $w_{0}=1, w_{1}=0, w_{2}=1$ and $w_{m}=0$ for all $m>2$. Then, $d_{01}$ is computed as

$$
d_{01}=\left(w_{0} \cdot \frac{1}{2^{1}}+w_{1} \cdot \frac{1}{2^{2}}+w_{2} \cdot \frac{1}{2^{3}}+\mathbf{0}\right)=\left(1 \cdot \frac{1}{2}+0 \cdot \frac{1}{4}+1 \cdot \frac{1}{8}\right)=\frac{5}{8}
$$

and the interval $\mathcal{I}_{01}$ is of radius $1 /\left(2^{1+2}\right)=1 / 8$ centered at $d_{01}=5 / 8$. In particular, we have $\mathcal{I}_{01}=[1 / 2,3 / 4]=[5 / 8-1 / 8,5 / 8+1 / 8]$.

For $w \in\{0,1\}^{*}$, we call the interval $\mathcal{I}_{w}$ constructed as above the standard dyadic interval at address $w$ (or "the standard dyadic interval centered at $d_{w}$ "), noting that these intervals are naturally in a one-one correspondence with the words in the monoid $\{0,1\}^{*}$ (we set $k=-1$ when $w=\varepsilon$, the empty word, so that we produce the interval $[0,1]$, that is, the closed interval of radius $1 / 2$ centred at $1 / 2)$.

To obtain the circle as a quotient of Cantor space we add one further identification, that is, we identify the point $\ldots 000=\overline{0} 0$ with the point $\ldots 111=\overline{1} 1$, noting that this simply identifies the real numbers 0 and 1 from the interval $I$.

When working in the unit interval, we will mostly use the real number parameterisation of points, but sometimes it is convenient to name a point by one of its names arising from the map $q^{-1}$. Similarly, for points on the circle, we will use either the parameterisation arising from the quotient map $I \rightarrow I /(0 \sim 1)=\mathbb{R} / \mathbb{Z}$ (this is equivalent to applying the map $p: I \rightarrow \mathbb{S}^{1}$ given by $t \mapsto e^{2 \pi i t}$ where we consider $\mathbb{S}^{1}$ as the unit circle in the complex plane) or, we will use the parameterisation arising from the map $q \cdot p: \mathfrak{C} \rightarrow \mathbb{S}^{1}$, where a point on the circle is referred to by one of its preimage left-infinite strings under the map $q \cdot p$.

Our group elements will act on the right, and induce permutations of the underlying sets of the spaces under consideration. We establish some notation for our context. Let $Y$ be a set. We will use the notation $\operatorname{Sym}(Y)$ for the group of bijections from $Y$ to itself. For any element $g \in \operatorname{Sym}(Y)$ we define the support of $g$, written $\operatorname{supt}(g)$, as the set

$$
\operatorname{supt}(g):=\{y \in Y: y g \neq y\},
$$


that is, the set of points moved by $g$. In keeping with our right-actions notation, if $g, h \in \operatorname{Sym}(Y)$, then the conjugate of $g$ by $h$, denoted $g^{h}$, is the map $h^{-1} g h$. That is, we apply $h^{-1}$, then $g$, and finally $h$ again. We then obtain the following standard lemma from the theory of permutation groups.

Lemma 2 Let $Y$ be a set, and $g, h \in \operatorname{Sym}(Y)$. We have

$$
\operatorname{supt}\left(g^{h}\right)=\operatorname{supt}(g) h \text {. }
$$

In particular, the support of $g^{h}$ is the image of the support of $g$ under the function $h$.

\section{The R. Thompson groups $F<T<V$}

The Thompson groups $F<T<V$ are groups of homeomorphisms which have been well studied. In this note, we generally take $F, T$, and $V$ as each being groups of homeomorphisms of the Cantor space $\mathfrak{C}$.

\subsection{Describing elements of $F, T$, and $V$}

For two words $w_{1}, w_{2} \in\{0,1\}^{*}$ with $\left|w_{1}\right|>0$ and $\left|w_{2}\right|>0$ we define the cone map $\phi_{w_{1}, w_{2}}: \mathfrak{C} w_{1} \rightarrow \mathfrak{C} w_{2}$ by the rule $\overleftarrow{x} w_{1} \mapsto \overleftarrow{x} w_{2}$, for each point $\overleftarrow{x}$ of $\mathfrak{C}$. It is immediate that this map is a homeomorphism from the Cantor space $\mathfrak{C} w_{1}$ to the Cantor space $\mathfrak{C} w_{2}$. Note that the map $\phi_{w_{1}, w_{2}}$ induces a map $\mathcal{I}_{w_{1}} \rightarrow \mathcal{I}_{w_{2}}$ which is a restriction of an affine map on the reals $\mathbb{R}$, and for this reason we might refer to $\phi_{w_{1}, w_{2}}$ as an "affine map" between the two subspaces of our larger Cantor space $\mathfrak{C}$. Note further that any such cone map $\phi_{w_{1}, w_{2}}$ is not just a homeomorphism from its domain to its range but also that it has many extensions to homeomorphisms from $\mathfrak{C} \rightarrow \mathfrak{C}$, and we can think of $\phi_{w_{1}, w_{2}}$ as being a subset of a larger (if $w_{1} \neq$ $\varepsilon \neq w_{2}$ ) function from $\mathfrak{C}$ to $\mathfrak{C}$ (which we in turn consider as a subset of $\mathfrak{C} \times \mathfrak{C}$ ).

We are now in a position to define the R. Thompson groups $F<T<V$.

An element $g \in \operatorname{Homeo}(\mathfrak{C})$ is an element of $V$ if and only if we can write $g$ as a prefix replacement map, as follows.

The element $g$ is a prefix replacement map if and only if it admits some natural number $n>1$, two complete antichains $D=\left\{a_{1}, a_{2}, \ldots, a_{n}\right\}$ and $R=$ $\left\{r_{1}, r_{2}, \ldots, r_{n}\right\}$ for $\{0,1\}^{*}$, and a bijection $\sigma: D \rightarrow R$, so that when restricted to any cone $\mathfrak{C} a_{i}$ (for valid index $i$ ), the map $g$ restricts and co-restricts to the cone map $\phi_{a_{1}, a_{1} \cdot \sigma}$. In this context, we will write

$$
g=\left(\left\{a_{1}, a_{2}, \ldots, a_{n}\right\},\left\{r_{1}, r_{2}, \ldots, r_{n}\right\}, \sigma\right) .
$$

We observe in passing that any element of $V$ admits infinitely many distinct prefix replacement maps representing it, but it is a standard exercise in R. Thompson theory that there is a unique, minimal prefix-map representation of $v$.

We observe that any complete antichain $\left\{a_{1}, a_{2}, \ldots, a_{n}\right\}$ for $\{0,1\}^{*}$ admits a natural left-to-right ordering $\prec$ induced from the arrangement of the addresses $a_{i}$ 
on the tree (this is simply the dictionary order, when we take $0 \prec 1$ and read our strings from right to left). An element $g \in V$ is in the subgroup $F$ if and only if, when expressed as a prefix replacement map, the permutation $\sigma$ preserves the ordering $\prec$. An element $g \in V$ is in the subgroup $T$ if and only if, when expressed as a prefix replacement map, the permution $\sigma$ preserves the ordering $\prec$ up to some cyclic rotation. It is then a standard exercise that elements of $F$ induce homeomorphisms of $I$ through the quotient map $q$ which are piecewise affine, respect the dyadic rationals, and where all slopes are powers of two and all breaks in slope occur over dyadic rationals. Similarly, it is a standard exercise that elements of $T$ induce homeomorphisms of $\mathbb{S}^{1}$ through the quotient $q \cdot p$ which are piecewise affine, respect the dyadic rationals, and where all slopes are powers of two and all breaks in slope occur over dyadic rationals.

A standard introductory reference for the general theory of the $\mathrm{R}$. Thompson groups $F, T$, and $V$ is the paper [6].

\subsection{The element family $\mathcal{X}$}

We now single out a family

$$
\mathcal{X}:=\left\{x_{w}: w \in\{0,1\}^{*}\right\}
$$

of elements of $V$ of specific interest to our discussion.

Given a word $w \in\{0,1\}^{*}$, we specify the element $x_{w}$ as the element of $V$ which acts as the identity over the complement of the cone $\mathfrak{C} w$, and on the cone $\mathfrak{C} w$, acts according to the prefix map specified below (we only express the actual prefix substitutions here):

$$
x_{w}:=\left\{\begin{aligned}
00^{\wedge} w & \mapsto 0^{\wedge} w \\
10^{\wedge} w & \mapsto 01^{\wedge} w \\
1^{\wedge} w & \mapsto 11^{\wedge} w
\end{aligned}\right.
$$

In particular, the element $x_{w}$ is the extension of the partial function

$$
\phi_{00^{\wedge} w, 0^{\wedge} w} \sqcup \phi_{10^{\wedge} w, 01^{\wedge} w} \sqcup \phi_{1{ }^{\wedge} w, 11^{\wedge} w}
$$

by the identity map away from the cone $\mathfrak{C} w$.

Note that it is easy to extend the set $\left\{00^{\wedge} w, 01^{\wedge} w, 1^{\wedge} w\right\}$ to a complete antichain $\left\{a_{1}, a_{2}, \ldots, a_{k-1}, 00^{\wedge} w, 10^{\wedge} w, 1^{\wedge} w\right\}$ for $\{0,1\}^{*}$ where $|w|=k$, and that in this case $\left\{a_{1}, a_{2}, \ldots, a_{k-1}, 0^{\wedge} w, 01^{\wedge} w, 11^{\wedge} w\right\}$ is also a complete antichain for $\{0,1\}^{*}$ (the set of addresses $\left\{a_{i}: 1 \leq i \leq k-1\right\}$ represents the minimal set of addresses one can use so that $\left\{a_{i}: i \in 1 \leq i \leq k-1\right\} \cup\{w\}$ is a complete antichain). Our map $x_{w}$ acts as cone maps on each of the cones at the set of addresses $\left\{00^{\wedge} w, 01^{\wedge} w, 1^{\wedge} w\right\}$, and otherwise takes each cone $\mathfrak{C} a_{i}$ to itself with the identity map.

It is easy to see that $\mathcal{X} \subset F$ so also $\mathcal{X} \subset T$ and $\mathcal{X} \subset V$. The figure below depicts the graphs of $x_{\varepsilon}$ and $x_{10}$ as homeomorphisms of $I$ as examples. 

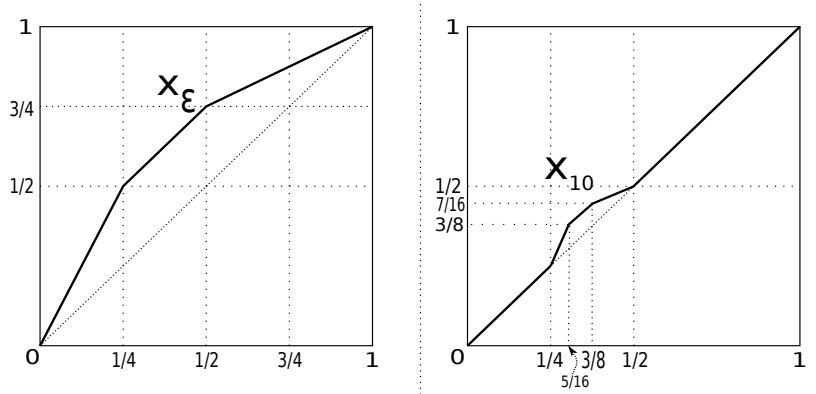

Fig. 2. The elements $x_{\varepsilon}$ and $x_{10}$.

\subsection{The element family $\mathcal{G}$}

For each natural $n \in \mathbb{N}$, set $g_{n}:=x_{(10)^{n}}$. The set $\mathcal{G}:=\left\{g_{i}: i \in \mathbb{N}\right\}$ will be our second family of elements of $F$ of interest. Note that we use $\mathbb{N}$ to represent the natural numbers, which we take to be the non-negative integers.

We observe that Figure 2 also depicts $g_{0}$ and $g_{1}$ since $g_{0}=x_{\varepsilon}$ and $g_{1}=x_{10}$.

\section{Realising ${ }^{\infty}(\mathbb{Z} \prec \mathbb{Z})$ in $F$}

Set $E:=\langle\mathcal{G}\rangle \leq F \leq T \leq V$. For each index $n$, consider the group

$$
W_{n}:=\left\langle\left\{g_{i}: i \in \mathbb{N}, i<n\right\}\right\rangle,
$$

where for clarity we specify $W_{0}=\left\{1_{V}\right\}$. It is immediate that $W_{m} \leq W_{n}$ when $m<n$. Direct calculation shows that $\operatorname{supt}\left(g_{n}\right) \cap \operatorname{supt}\left(g_{n}^{g_{m}}\right)=\emptyset$ whenever $m<n$ (the content of the following lemma, which is not hard to prove). Specifically, following the arguments of $[2,3]$ for natural index $n$ we have $W_{n} \cong(\ldots((\mathbb{Z} \imath \mathbb{Z})$ ? $\mathbb{Z}) \ldots(\mathbb{Z}) \succ \mathbb{Z}$ (with $n$ appearances of $\mathbb{Z}$ in this expression), which is a solvable group of derived length $n$. We immediately obtain $E \cong \infty(\mathbb{Z} \imath \mathbb{Z})=\ldots \imath \mathbb{Z})(\mathbb{Z}) \imath \mathbb{Z}$, as described in detail in [4]. As $E$ admits a decomposition as a direct union of the solvable groups $W_{n}$, we obtain that $E$ is elementary amenable (see Chou's paper [7] for details on the class of elementary amenable groups).

For what follows, set $G_{m, n}:=\left\langle g_{m}, g_{n}\right\rangle$ for all natural numbers $m<n$.

Lemma 3 Let $m<n$ be two natural numbers. We have

1. there is an isomorphism $G_{m, n} \cong G_{0,(n-m)}$ which is induced by a restriction map followed by a topological conjugacy,

2. $\operatorname{supt}\left(g_{n}\right) \cap \operatorname{supt}\left(g_{n}^{g_{m}}\right)=\emptyset$, and therefore

3. $G_{m, n} \cong \mathbb{Z} \imath \mathbb{Z}$.

Proof. We first prove Point (1) that there is an isomorphism $G_{m, n}:=\left\langle g_{m}, g_{n}\right\rangle \cong$ $\left\langle g_{0}, g_{n-m}\right\rangle=G_{0,(n-m)}$ which is induced by a restriction map followed by a topological conjugacy. 
To see this point, first observe that both of the elements $g_{n}$ and $g_{m}$ are supported wholly in the cone $\mathfrak{C}(10)^{m}$, so, the restriction of the maps $g_{n}$ and $g_{m}$ to the cone $\mathfrak{C}(10)^{m}$ results in an isomorphism of groups between the homeomorphism group $G_{m, n}=\left\langle g_{m}, g_{n}\right\rangle$, which acts on the Cantor space $\mathfrak{C}$, to a homeomorphism group $\widehat{G}_{m, n}=\left\langle\widehat{g}_{m}, \widehat{g}_{n}\right\rangle$ (these generators being the restrictions of the generators $g_{m}$ and $g_{n}$ respectively), so that the group $\widehat{G}_{m, n}$ is a group of homeomorphisms of the Cantor space $\mathfrak{C}(10)^{m}$. Now, the homeomorphism $\theta_{m}: \mathfrak{C}(10)^{m} \rightarrow \mathfrak{C}$ which is induced by deleting the prefix $(10)^{m}$ from all points in the Cantor space $\mathfrak{C}(10)^{m}$ provides a topological conjugacy which induces an isomorphism from the group $\widehat{G}_{m, n}$ to the group $G_{0, n-m}=\left\langle g_{0}, g_{(n-m)}\right\rangle$, as the reader can check that the image of the $\widehat{g}_{m}$ is the element $g_{0}$ and the image of $\widehat{g}_{(n)}$ is the element $g_{(n-m)}$ under this topological conjugacy.

For Point (2), we observe that the restrictions applied in the argument for Point (1) only removed areas from the domain of the elements $g_{m}$ and $g_{n}$ where these elements already acted as the identity. Therefore the support of $g_{n}$ and of $g_{n}^{g_{m}}$ will be disjoint if and only if the supports of the elements $g_{(n-m)}$ and of $g_{(n-m)}^{g_{0}}$ are disjoint. In particular, we have our result if we prove that for any positive integer $k$, we have $\operatorname{supt}\left(g_{k}\right) \cap \operatorname{supt}\left(g_{k}^{g_{0}}\right)=\emptyset$.

However, $g_{0}=x_{\varepsilon}$, which acts over the cone $\mathfrak{C} 10$ as a cone map, affinely taking the cone $\mathfrak{C} 10$ rightward to the cone $\mathfrak{C} 01$ by the prefix substitution $10 \mapsto 01$. Now, the support of $g_{k}$ is contained in the cone $\mathfrak{C}(10)^{k}$, a subset of the cone $\mathfrak{C}(10)$. Direct calculation now shows that the cone $\mathfrak{C}(10)^{k}$ is carried affinely to the cone $\mathfrak{C}(10)^{k-1}(01)$ by $g_{0}$, so Lemma 2 implies our result. We note in passing that we have shown that $g_{k}^{g_{0}}=x_{(10)^{k-1} 01}$, or more specifically, that $x_{(01)^{k}}^{x_{\varepsilon}}=x_{(10)^{k-1} 01}$, since our conjugator acted affinely.

For Point (3), recall Section 1.2.1 of [2], where an argument is given that two elements $\alpha_{1}$ and $\alpha_{2}$ of $F$ generate a group isomorphic to $\mathbb{Z} \imath \mathbb{Z}$, with the element $\alpha_{1}$ generating the top group of the wreath product. It happens that the element $\alpha_{1}$ of that paper is the element we call $x_{\varepsilon}$ here, while the element $\alpha_{2}$ is the element we call $x_{10}$ here. The proof of Section 1.2.1 essentially relies on only three facts: 1) the support of $\alpha_{2}$ is contained in the support of $\left.\alpha_{1}, 2\right)$ every point in the support of $\alpha_{1}$ is on an infinite orbit under the action of $\left\langle\alpha_{1}\right\rangle$, and 3) the support of $\alpha_{2}$ is moved entirely off of itself by $\alpha_{1}$. In our case with $g_{m}$ and $g_{n}$, we again have these three conditions (with $g_{m}$ playing the role of $\alpha_{1}$ ), so we have our claimed Point (3).

The discussion above indicates the following lemma.

Lemma 4 Let $v \in V$. There are $\left\{a_{1}, a_{2}, \ldots, a_{n}\right\}$ and $\left\{b_{1}, b_{2}, \ldots, b_{n}\right\}$, minimal cardinality (finite) antichains, together with a bijection $\sigma$ between them, so that $v$ can be described as the prefix replacement map

$$
v=\left(\left\{a_{1}, a_{2}, \ldots, a_{n}\right\},\left\{b_{1}, b_{2}, \ldots, b_{n}\right\}, \sigma\right) .
$$

If $w, u \in\{0,1\}^{*}$ so that $w=u^{\wedge} a_{i}$ for some $i$, then $x_{w}^{v}=x_{u^{\wedge}\left(a_{i} \sigma\right)}$. 
Proof. By definition, we have $x_{w}^{v}=v^{-1} x_{w} v$. By our assumptions $w$ has $a_{i}$ as a prefix, and we note that the initial map $v^{-1}$ restricts to a cone map from $\mathfrak{C}\left(a_{i} \sigma\right)$ to $\mathfrak{C} a_{i}$, that is, an affine map with image containing the support of $x_{w}$. The action of $x_{w}$ off the cone $\mathfrak{C} a_{i}$ is as the identity, and in general is as described in the definition of $x_{w}$ (it acts as a prefix replacement map, which modifies only the prefixes which begin with $w=u^{\wedge} a_{i}$, and these modifications appear in entries at indices larger than the length $|w|)$. Finally, $v$ acts on the cone $\mathfrak{C} a_{i}$ by affinely returning it to $\mathfrak{C}\left(a_{i} \sigma\right)$ as a cone map, (it simply transforms the prefix $a_{i}$ to the prefix $a_{i} \sigma$, and preserves all later entries (with index offset of size $\left|a_{i} \sigma\right|-\left|a_{i}\right|$ ) at larger indices, for any point in the Cantor space $\left.\mathfrak{C}\left(a_{i}\right)\right)$. Therefore, $x_{w}^{v}=x_{u^{\wedge}\left(a_{i} \sigma\right)}$.

\section{On partial actions}

The proof of Lemma 4 suggests the well-known fact that $V$ has a natural partial action on the addresses in $\{0,1\}^{*}$. Let $v \in V$ and suppose there is a minimal natural number $n$ and antichains $A=\left\{a_{1}, a_{2}, \ldots, a_{n}\right\}$ and $B=\left\{b_{1}, b_{2}, \ldots, b_{n}\right\}$ with a bijection $\sigma$ between them so that $v$ can be described as the prefix replacement map $v=(A, B, \sigma)$. The partial action of $v$ on $\{0,1\}^{*}$ is defined precisely on the set of words in $\{0,1\}^{*}$ which admit one of the $a_{i}$ as a prefix. Let us suppose $w \in\{0,1\}^{*}$ and $w=u^{\wedge} a_{i}$. We set $w \cdot v:=u^{\wedge}\left(a_{i} \sigma\right)$.

We can now re-express the result of Lemma 4 in terms of the partial action of $V$ on the set $\{0,1\}^{*}$.

Corollary 5 Let $v \in V$ and $w_{1}, w_{2} \in\{0,1\}^{*}$ so that $w_{1} \cdot v=w_{2}$ under the partial action of $V$ on $\{0,1\}^{*}$. If $u \in\{0,1\}^{*}$ then $x_{u^{\wedge} w_{1}}^{v}=x_{u^{\wedge} w_{2}}$.

That is, we see that the group $V$ admits a partial action on the set $\mathcal{X}$ which parallels its partial action on $\{0,1\}^{*}$. We now work to understand the action of $V$ on elements of the group $E$.

Our first step in understanding this partial action is to analyse a formal language.

\subsection{A regular language and an action}

Define the set $\mathcal{T} \subset\{0,1\}^{*}$ of tokens as follows:

$$
\mathcal{T}:=\left\{10^{k}, 01^{k}: k \in \mathbb{N}, k \neq 0\right\} .
$$

We build a formal language $\mathcal{W}$ over the alphabet $\{0,1\}$ as follows. The language $\mathcal{W}$ is the set of all words which decompose as $w=w_{j}{ }^{\wedge} w_{j-1} \wedge \ldots \wedge w_{1}$ for some natural $j$, where each $w_{i}$ is a token. The language $\mathcal{W}$ is actually a regular language, which is recognised by the automaton $\mathcal{A}$ depicted in Figure 3 . The state $q_{0}$ of $\mathcal{A}$ is both the start and accept state of $\mathcal{A}$. 


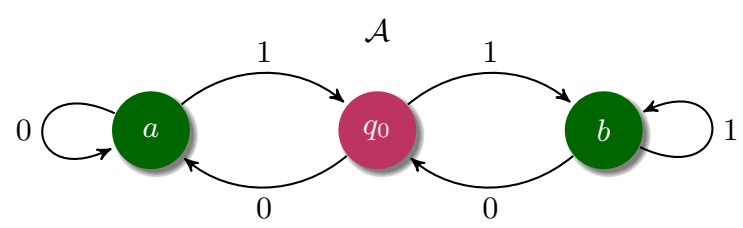

Fig. 3. The automaton $\mathcal{A}$ which accepts the language $\mathcal{W}$

Recall that an automaton is a finite directed edge-labelled graph with a subset of its set of states called the start states of the automaton, and another subset of its set of states called the accept states of the automaton. Then, the language accepted by the automaton is precisely the set of all finite-length words which arise as the concatenated edge-labels of some finite path in the automaton from a start state to an end state.

One can see that our formal language $\mathcal{W}$ is indeed the language accepted by $\mathcal{A}$; the paths which leave the state $q_{0}$ and then eventually return (exactly once) have labels of the form $10^{k}$ or $01^{k}$, for some non-zero nautral number $k$. In particular, the language accepted by $\mathcal{A}$ is precisely the language of words built by concatenating tokens from $\mathcal{T}$.

We now set some terminology describing the structure of elements of the language $\mathcal{W}$. Observe firstly that the decomposition of any word in $\mathcal{W}$ into a concatenation of tokens is unique. Therefore, for each $w \in \mathcal{W}$ we can define the token length of $w$ as the number of tokens in its decomposition as a concatenation of tokens. Note that we index these tokens from right to left: $w=w_{k} \wedge w_{k-1} \wedge \ldots \wedge w_{2} \wedge w_{1}$.

We now observe that the partial action of $V$ on $\{0,1\}^{*}$ restricts to an action of $\left\langle g_{0}\right\rangle$ on the set $\mathcal{T}$. Below, the proofs of Lemmas 6 and 8 follow by simple inductions on a basic calculation.

Lemma 6 For each integer $k$, the restriction of $g_{0}^{k}$ to the cone $\mathfrak{C} 10$ produces a cone map from the cone $\mathfrak{C} 10$ to the cone $\mathfrak{C} w_{k}$, where $w_{k}$ is given by the formula below:

$$
w_{k}=10 \cdot g_{0}^{k}=\left\{\begin{array}{cc}
10^{|k-1|} & k \leq 0 \\
01^{k} & k>0
\end{array}\right.
$$

Proof. This proof is a simple induction. Recall that $g_{0}=x_{\varepsilon}$.

If $k=0$ we observe that our formula works as $g_{0}^{0}=1_{V}$, which maps the cone $\mathfrak{C} 10$ to the cone $\mathfrak{C} 10$ by the identity map, which is a cone map. If $k=1$ then $g_{0}$ takes $\mathfrak{C} 10$ to $\mathfrak{C} 01$ as a cone map, in accordance with the definiton of $x_{\varepsilon}$. For all $k \geq 2, g_{0}^{k}$ acts as $x_{\varepsilon} \cdot x_{\varepsilon}^{k-1}$, so first as a cone map from $\mathfrak{C} 10$ to $\mathfrak{C} 01$, and then it will continue to act as $x_{\varepsilon}^{k-1}$ on this resulting cone. However, the cone $\mathfrak{C} 01$ is contained in the cone $\mathfrak{C} 1$, and so the prefix replacement of $x_{\varepsilon}$ here replaces the initial prefix 1 with the prefix 11, and this process repeats so inductively we have our desired result for all integers $k \geq 0$. For negative integers $k$, the argument 
follows as $x_{\varepsilon}^{-1}$ replaces the prefix 0 with the prefix 00 , and so inductively, the cone at 10 is carried by a cone map to the cone at $10^{|k-1|}$ by $x_{\varepsilon}^{k}$.

A translation of the above result is as follows.

Corollary 7 The partial action of $V$ on the set $\{0,1\}^{*}$ restricts to a free, transitive action of $\left\langle g_{0}\right\rangle$ on the set $\mathcal{T}$ of tokens.

The following lemma simply extends the result of Lemma 6 .

Lemma 8 For each integer $k$ and natural number $i$, the restriction of $g_{i}^{k}$ to the cone $\mathfrak{C}(10)^{i+1}$ produces a cone map from the cone $\mathfrak{C}(10)^{i+1}$ to the cone $\mathfrak{C} w_{i, k}$, where $w_{i, k}$ is given by the formula below:

$$
w_{i, k}=(10)^{i+1} \cdot g_{i}^{k}=\left\{\begin{array}{cc}
10^{|k-1|}(10)^{i} & k \leq 0 \\
01^{k}(10)^{i} & k>0 .
\end{array}\right.
$$

Proof. The proof is similar to the proof of Lemma $6 ; g_{i}=x_{(10)^{i}}$ acts as the identity off of the cone $\mathfrak{C}(10)^{i}$, and acts on the cone $\mathfrak{C}(10)^{i}$ in the same way that $g_{0}$ acts on the cone $\mathfrak{C} \varepsilon=\mathfrak{C}$ (this is essentially the content of the proof of Lemma $3(1)$. That is, the prefix $(10)^{i}$ is fixed by all powers of $g_{i}$, but the word $(10)^{i+1}$ is changed by $g_{i}$ on the final token " 10 " (the " 0 " at index $2 i$ and the " 1 " at index $2 i+1)$.

Lemma 8 has the following related corollary.

Corollary 9 Let $i$ be a natural number. The partial action of $V$ on the set $\{0,1\}^{*}$ restricts to a transitive and free action of $\left\langle g_{i}\right\rangle$ on the set of words $\left\{t^{\wedge}(10)^{i}: t \in\right.$ $\mathcal{T}\}$.

\section{$6 \quad$ Visiting the family $\mathcal{X}$}

We now discuss the intersection of the group $E=\langle\mathcal{G}\rangle$ with the family $\mathcal{X}$.

Lemma 10 Let $w \in \mathcal{W}$, and $k \in \mathbb{N}$ so that $w$ has token decomposition $w=$ $w_{k}{ }^{\wedge} w_{k-1}{ }^{\wedge} \ldots{ }^{\wedge} w_{2}{ }^{\wedge} w_{1}$. For each token $w_{i}$, let $j_{i}$ be the integer so that $10 \cdot x_{\varepsilon}^{j_{i}}=w_{i}$ and also, recall that $x_{\varepsilon}=g_{0}$. If we set $\theta_{w}$ to be the product

$$
\theta_{w}:=g_{k-1}^{j_{k}} g_{k-2}^{j_{k-1}} \cdots g_{1}^{j_{2}} g_{0}^{j_{1}}
$$

then we have

$$
x_{w}=g_{k}^{\theta_{w}} .
$$

Proof. One constructs $\theta$ by modifying the prefix $(10)^{k}$ to the prefix $w$ by acting on one token at a time, starting with the leftmost token (the $k^{\text {th }}$ token), and then working to the first token $w_{1}$. Progressively, each term in the product decompositon of $\theta$ acts on a cone containing the impact of the previous terms which have acted, the actions stack to create the following sequence of prefixes for the locations of the actions of the (partially) conjugated versions of $x_{(10)^{k}}$. 


$$
\begin{aligned}
& (10)^{k} \mapsto \\
& w_{k}(10)^{k-1} \mapsto \\
& w_{k} w_{k-1}(10)^{k-2} \mapsto \\
& \ldots \\
& w_{k} w_{k-1} \ldots w_{2} 10 \mapsto \\
& w_{k} w_{k-1} \ldots w_{2} w_{1} .
\end{aligned}
$$

We therefore have the following corollary.

Corollary 11 Let $\mathcal{X}_{\mathcal{W}}:=\left\{x_{w}: w \in \mathcal{W}\right\}$. Then $\mathcal{X}_{\mathcal{W}} \subset E$.

We now consider a special subset of $\mathcal{W}$. Set

$$
\mathcal{S}:=\left\{100^{\wedge} w, 011^{\wedge} w: w \in\{0,1\}^{*}\right\}
$$

Lemma 12 The set $\mathcal{S}$ is a subset of $\mathcal{W}$.

Proof. The automaton $\mathcal{A}$ of Figure 3 has further properties not mentioned previously; it is highly connected and synchronizing. These properties together mean that given any particular state (let us say $q_{0}$ ), there is a non-empty set of synchronizing words $W_{q_{0}}$ associated with $q_{0}$ so that, starting from any particular state $s$ of the automaton and following a path labelled by any word in $W_{q_{0}}$, perforce, one will be lead to the state $q_{0}$.

Note that the words 100 and 011 are synchronizing words for the state $q_{0}$; no matter what state one starts in, after following the path labelled by the word 100 from that state, or the path labelled by the word 011 from that state, one arrives in the state $q_{0}$ (recall that we are reading these words from right-to-left!).

Thus, if we have some general word $w$ and we append a suffix 011 or 100 to produce either $z=011^{\wedge} w$ or $z=100^{\wedge} w$ (that is, a general word $z$ in $\mathcal{S}$ ), then upon reading this resulting word on the automaton $\mathcal{A}$ starting from the start state $q_{0}$, we will return to $q_{0}$; our word $z$ is in the language $\mathcal{W}$ accepted by $\mathcal{A}$.

Below, we will actually be interested in the subset of $\mathcal{X}_{\mathcal{W}}$ where the words involved come from $\mathcal{S}$. Set

$$
\mathcal{X}_{S}:=\left\{x_{w}: w \in \mathcal{S}\right\}
$$

Corollary 13 The set $\mathcal{X}_{S}$ is a subset of the group E.

We now consider how $V$ interacts with the set $\mathcal{X}_{S}$ under conjugation. The following lemma follows quickly by an application of Corollary 5.

Lemma 14 Let $v \in V$. There is a natural number $n$ so that for all $w \in \mathcal{S}$ with $|w| \geq n$ there is $z \in S$ so that $x_{z}^{v}=x_{w}$.

Proof. Let us assume that we can represent $v$ by some prefix replacement map

$$
v=\left(\left\{a_{1}, a_{2}, \ldots, a_{m}\right\},\left\{b_{1}, b_{2}, \ldots, b_{m}\right\}, \sigma\right)
$$


where we assume that $n$ is at least three larger than the length of the largest string in the range antichain $\left\{b_{1}, b_{2}, \ldots, b_{m}\right\}$. We then set $b$ as the prefix of $w$ appearing in the set $\left\{b_{1}, b_{2}, \ldots, b_{m}\right\}$, and set $a=b \sigma^{-1} \in\left\{a_{1}, a_{2}, \ldots, a_{m}\right\}$. Then, $w=c^{\wedge} b$ where $c$ is some string of length at least three, and we have $\left(c^{\wedge} a\right) \cdot v=\left(c^{\wedge} b\right)=w$, so that, in particular, if we take $z:=c^{\wedge} a$, then Corollary 5 assures us that $x_{z}^{v}=x_{c^{\wedge} b}=x_{w}$.

But now, as the word $c$ has length at least three, we see that it must end with the string 100 or the string 011 (since $w$ has one of these two length three suffixes), and in particular, $z \in \mathcal{S}$.

Thus, we have found that all sufficiently long strings $w$ in $\mathcal{S}$ have that $x_{w}$ is the conjugate image of $x_{z}$ under $v$, for $z$ another string in $\mathcal{S}$.

Proof of Theorem 1:

Proof. The group $E$ of this note is infinite and amenable. We can further see that for any finite set $C:=\left\{v_{1}, v_{2}, \ldots, v_{k}\right\} \subset V$, the elements $x_{w}$, for $w \in \mathcal{S}$ with $w$ long enough (given by some particular integer dependent on the set $C$ ), all appear in all of the groups $E^{v_{i}}$. In particular, the intersection

$$
\bigcap_{v_{i} \in C} E^{v_{i}}
$$

is an infinite set, so that $E$ is normalish in each of $F, T$, and $V$.

\section{References}

1. C. Bleak, An exploration of normalish subgroups of R. Thompson's groups $F$ and $T$, (submitted) arXiv:1603.01726 (2016), 1-4.

2. Collin Bleak, An algebraic classification of some solvable groups of homeomorphisms, J. Algebra 319 (2008), no. 4, 1368-1397. MR 2383051

3 . A geometric classification of some solvable groups of homeomorphisms, J. Lond. Math. Soc. (2) 78 (2008), no. 2, 352-372. MR 2439629

4. A minimal non-solvable group of homeomorphisms, Groups Geom. Dyn. 3 (2009), no. 1, 1-37. MR 2466019

5. Emmanuel Breuillard, Mehrdad Kalantar, Matthew Kennedy, and Narutaka Ozawa, $C^{*}$-simplicity and the unique trace property for discrete groups, Publ. Math. Inst. Hautes Études Sci. 126 (2017), 35-71. MR 3735864

6. J. W. Cannon, W. J. Floyd, and W. R. Parry, Introductory notes on Richard Thompson's groups, Enseign. Math. (2) 42 (1996), no. 3-4, 215-256.

7. Ching Chou, Elementary amenable groups, Illinois J. Math. 24 (1980), no. 3, 396-407.

8. Uffe Haagerup and Kristian Knudsen Olesen, Non-inner amenability of the Thompson groups $T$ and $V$, J. Funct. Anal. 272 (2017), no. 11, 4838-4852. MR 3630641

9. M. Kennedy, Characterisations of $C^{*}$ simplicity, (submitted). arxiv:1509.01870v3 (2015), 1-16.

10. Adrien Le Boudec and Nicolás Matte Bon, Subgroup dynamics and $C^{*}$ simplicity of groups of homeomorphisms, Ann. Sci. Éc. Norm. Supér. (4) 51 (2018), no. 3, 557-602. MR 3831032 